\title{
Endodontic Periapical Disease: A Virus-Based Perspective
}

\author{
Mohammad Sabeti $^{1 *}$, Kory J Golchert ${ }^{1}$, Neveed Shirgill ${ }^{1}$, Aleksandar Jakovljevic ${ }^{2}$, Miroslav Andric ${ }^{3}$ and \\ Jelena Milasin ${ }^{4}$ \\ ${ }^{1}$ Department of Endodontics, School of Dentistry, University of California, San Francisco, USA \\ ${ }^{2}$ Department of Pathophysiology, School of Dental Medicine, University of Belgrade, Serbia \\ ${ }^{3}$ Department of Oral Surgery, School of Dental Medicine, University of Belgrade, Serbia \\ ${ }^{4}$ Department of Human Genetics, School of Dental Medicine, University of Belgrade, Serbia
}

*Corresponding author: Mohammad Sabeti, Department of Preventative and Restorative Dental Science, School of Dentistry, University of California, San Francisco, California, USA.

Received Date: June 29, 2019

Published Date: July 11, 2019

\begin{abstract}
There is a need for updating the current treatment modalities for periapical lesions of endodontic origin to reflect the contributions and potential morbidity from herpesvirus infection. Herpesviruses, particularly human cytomegalovirus and Epstein-Barr virus, play an important role in the symptomatic periapical lesion. Indeed, periapical lesions may remain stable and silent until they become symptomatic, possibly triggered by the activation of latent herpesviruses within the lesion itself. Given the potential for grave systemic conditions associated with herpesvirus infection, early detection and treatment decisions for periapical lesions by the dentist may positively affect a patient's overall health; especially for the immunocompromised patient.
\end{abstract}

Keywords: Endodontic disease; Apical periodontitis; Herpesviruses; Classification

Abbreviations: RCT: Root canal treatment; HHV: Human Herpesvirus; EBV: Epstein-Barr Virus; HCMV: Human Cytomegalovirus; CBCT: ConeBeam Computed Tomography

\section{Introduction}

\section{Apical disease etiopathogenesis}

A periapical pathogenic infection exhibits complex microbial ecologies. There is a predominance of obligate anaerobic bacteria in the necrotic pulp, and a close correlation with black-pigmented anaerobic rods in acute periapical inflammation [1]. Many endodontopathic black-pigmented species such as P. gingivalis, P. nigrescens, and P. intermedia have been observed in extra-radicular lesions [2] and strong correlations have been observed with the species P. endodontalis, S. sputigena, and Peptostreptococcus micros [3]. In contrast, there is negative or no correlation with Canocytophaga ochraces, Veillonella parvula and other endodontopathic species [3].

Some patients have apical lesions and defective root canal treatments which are detected by routine exams or have been asymptomatic for years. There have been many varying hypotheses on the etiopathogenesis of apical pathosis that include both bacterial and host factors [2]. It is not clear, however, how a stable apical pathosis becomes unstable. For example, how do bacteria that have established a permanent residence in necrotic teeth and voided root canal fillings with microbial presence become unstable? As well, how do asymptomatic teeth with apical lesions become unstable?

Besides the various species of bacteria that are pathognomonic of acute periapical infections another major factor, the contribution of viral infection, should also be considered [4]. In particular, the herpesvirus, with its cycle of inactivation and activation may explain the episodic nature of periapical disease. Ferreira et al showed in two separate studies that exudative samples from acute apical abscesses most often contained human herpesvirus-8 (HHV8; $48 \%$ and $54.5 \%$ of samples] [5,6]. Guilherme et al found that saliva samples from patients with root canals contained HHV-8 in $84 \%$ of successful root canals, and $89 \%$ of failing root canals [7]. The Epstein-Barr virus (EBV) was found in $72.5 \%$ of apical periodontal 
lesions in a subpopulation of Hungarians [8]. Additionally, these same studies identified the presence of several other herpesviruses in some of their samples including varicella zoster virus and human cytomegalovirus (HCMV). Jakovljevic et al. reported that EBV EB nuclear antigen type I and HCMV glycoprotein B (gB) type 2 are the most frequently detected genotypes in apical periodontitis in a Serbian population [9]. While in its latent state, the inactive virus may correspond to extended periods of dormant periapical disease progression, whereas the active virus may be associated with rapid periapical tissue breakdown. A vicious cycle between the varying bacterial composition of the biofilm in the dentinal tubules or accessory canals, along with herpesvirus coinfection, offers a viable explanation for the conversion of a stable apical pathosis into an unstable state.

The overgrowth of pathogenic microorganisms alongside consequent immune impairment and other virulence factors make herpesvirus a potent feature in the formation of periapical pathosis [1]. The presence of HCMV or EBV is strongly connected with increased levels of P. intermedia, A. actinomycetecomitans, $\mathrm{T}$. forsythensis and P. gingivalis bacterial species [10]. From a sample of 58 teeth with apical periodontitis, $75.9 \%$ contained EBV and were significantly associated with the presence of the pro-inflammatory and osteoclastic mediator tumor necrosis factor-alpha [11]. HCMV glycoprotein $\mathrm{gB}$ and several surface proteins of EBV, including the glycoprotein gp350, latent membrane protein 1, and nuclear antigen 2 are implicated in the strong cellular signaling responses of the host [12]. Nonappearance of herpesviral factors may be the reason for extended periapical lesion dormancy and stability. When herpesvirus is not present, or the viral vectors are not reactivated, the periapical disease progression is halted. This significant factor may hold the key for periapical disease treatment [13].

In immunocompetent and immunocompromised individuals, the innate immune response to initial herpesvirus infection is activated through recognition of the virus by toll-like receptors [14]. As the viral infection enters latency, reactivation of the virus is combatted primarily by adaptive immune responses [15]. A diminished immune response has been observed in mice co-infected with P. gingivalis and cytomegalovirus. This has been attributed to a down-regulation of T-cell receptors from bacterial antigens [16]. The importance of herpesvirus associations in periapical pathosis has been established [17,18]. In addition, a significant link of bacterial co-infection with herpesviral vectors and a tendency for a more severe and progressive form of the disease has been shown [4]. The results of these studies indicate an undefined role of herpesvirus factors as another causative agent in the impaired host immune response to endodontic infection. Furthermore, the effect of bacterial and herpesvirus co-infection in immunocompromised individuals warrants further study. This implies a direct need for stricter prophylactic treatments for endodontic diseases that increase patient compliance and care.

Associated host responses can also explain most of the variations in the clinical manifestations of endodontic pathosis $[17,18]$.
However, there is not a clear guideline for the recommendation of treatment for asymptomatic periapical lesions. Therefore, should we consider any tooth presenting with a periapical lesion as a diseased tooth? The current endodontic diagnosis rubric does not formulate a specific treatment, nor help in the understanding of how an unstable lesion becomes symptomatic. In addition, biofilms alone cannot explain the progression of the disease. Herpesvirus activation by impairment of cell-mediated immunity should be included as a potential trigger point for progressive and persistent apical disease.

Therefore, this article is aimed to highlight the need for an update to current terminology and related therapy for the classification and diagnosis of periapical disease of endodontic origin based on viral status. In addition to the well-known bacterial contribution to periapical disease, many recent studies have shown that viruses, particularly herpesviruses, may play an important role. Given the potential serious effect herpesviruses have on general health, especially in immunocompromised individuals, the dental clinician is uniquely situated for both the detection of the virus and the prevention of systemic sequelae from herpesvirus infections.

\section{Discussion}

\section{Endodontic diagnostics and classification}

A big challenge in endodontics is how to approach the apparent under-diagnosis of periapical disease. Are any low-density findings in a CBCT image associated with the periapical area of a tooth alone justification for treatment? Because very early lesions may not show any radiographic changes, diagnosis of these lesions relies on the clinical symptoms of pulpitis or necrosis, and the method of screening for the symptoms.

The current endodontic diagnosis rubric does not help us to understand the pathophysiology and timespan of the disease from its initiation to the appearance of clinical signs and symptoms. Acute apical disease is characterized by swelling and formation of pus, while chronic apical disease is the state with sinus tract formations [19]. However, these terms contain no information that can guide us to a specific etiology to formulate a therapeutic guide. The current classification and disease features may help us to understand when a disease is stable, but do not provide any information to guide us to understand an unstable lesion. Variables such as etiology, disease activity and degree of apical tissue destruction should be used in formulating a new classification system in order to guide us to better treatment options with better healing rates.

\section{Endodontic epidemiology}

Periapical pathosis is a compound condition having multiple factors, arising from either infection of necrotic pulp, or a failed endodontic treatment. It occurs when the bacterial infection within the pulp and canal space elicits an inflammatory response around the apex of the root. This inflammatory process can also be aggravated due to herpesviral vectors.

The prevalence estimates of endodontic lesions depend on the case definition of either acute or chronic conditions and the 
radiographic features of periapical inflammatory lesions, which vary depending on the time course of the lesion. It affects a significant proportion of people in different populations. Numerous studies have shown prevalence estimates for apical periodontitis in root filled teeth from many distinct geographical areas. For example, in a Danish population of adults 18 years or older $20 \%$ of root filled teeth had apical periodontitis [20], while $64.5 \%$ of root filled teeth had apical periodontitis in a Spanish population of adults 18 years or older [21]. The range of prevalence of apical periodontitis across geographical areas varies widely and is confounded by differing ages within each population. Indeed, the studies investigated an adult population over 18 years of age, but apical periodontitis prevalence $(44.3 \%)$ in root filled teeth in a Canadian population was limited to 25-40-year old [22].

The initial progression of the apical disease process is aided by herpesviral infection, although this cannot be detected by conventional clinical and radiographic methods [23]. This initial infection creates a synergistic relationship with the microbial organisms within the biofilm and the initiation of periapical infections [24]. Biofilm acids diffuse below the surface and demineralize the more soluble subsurface of tooth structure. This process leads to lymphocyte and memory B-cell infiltration [16]. In addition to the $\mathrm{T}$ cell and B-cell infiltrates, macrophages also proliferate. The inflammatory process is further aided by herpesviral vectors which can cause further inflammation and dissolution $[12,25]$.

Severe apical periodontitis is due to specific bacterial species and a destructive immune response, which is identical to active herpesvirus infections. The body of research suggests a role for herpesvirus in the development of periapical pathosis. Herpesvirus activation may lead to breakdown of the periodontal tissue and lead to severe periapical periodontitis [26]. Furthermore, in cases of latency of the herpesvirus, severe apical pathosis may not be seen. As CBCT has become more widely used in endodontic diagnosis, many asymptomatic periapical lesions have likely gone previously undetected $[27,28]$. These may represent inactive endodontic lesions with latent herpesvirus infection. The herpesvirus and the microbiota of periapical disease form a commensal relationship and may work in conjunction with bacteria responsible for severe apical periodontitis and as such, herpesvirus may result in the formation of the periapical disease state. A potential causal relationship has been shown between EBV and anaerobic bacteria in symptomatic apical periodontitis [29]. Therefore, preventing herpesvirus activation by using antiviral medication may affect the degree of breakdown of the periapical tissue and the development of active apical disease. The intersection of genetics, infectious disease states, immunology and their role in periapical pathosis can be better understood when a clear connection between herpesvirus and apical disease is established.

Endodontic lesions occurred with particularly high prevalence in low-income and older individuals (30), though the concurrent number of people having both apical disease and herpesviral infection remains unknown. It has been noted that a high number of the population in developing countries may be infected with herpesvirus [31], and its role in the etiopathogenesis of apical pathosis cannot be ignored. The average worldwide prevalence of severe apical disease has been estimated to be markedly high, including countries with relatively little emphasis on periapical health care [32].

\section{Apical pathosis and systemic disease}

There is a possible link between periapical pathosis and systemic disease states although yet, no identifiable correlation between bacterial causes of apical periodontitis and systemic disease exist. Latent HCMV virus reactivation in apical disease poses a higher risk by impairment of immune system and susceptibility to diseases [33]. Many cancers have been associated with herpes viruses [4]. Latent HCMV virus reactivation also poses a higher risk of failure of organ transplants in immunocompromised patients [34]. HCMV has been linked to bone marrow transplant failures with significant mortality. Primary and reactivated HCMV infection is well known to become established in immunocompromised hosts, patients with AIDS [16]. It appears to be a possibility that the potential source of the virus is a latent infection in periapical lesions.

Systemic conditions, including but not limited to diabetes mellitus and cardiovascular disease serve as modifying factors which limit the survivability of teeth treated endodontically [35]. These systemic modifying factors act to disrupt the immune response and as a result may activate latent herpesviruses, thus affecting the healing ability of the pulp and periapical tissues. Systemic disease states influence the creation of apical pathosis by their action on healing and the host immune response and therefore influence treatment outcomes of apical conditions. These systemic factors show correlation, and therefore must be included as diagnostic factors in periapical disease. Care must be taken, however, when associating systemic disease states with apical conditions since the literature does not currently support an absolute correlation of systemic disease states to endodontic pathosis [36]. If strong correlations between infection by herpes virus and apical pathosis can be elucidated, diagnosis and treatment protocols would improve leading to effective treatment for both the herpesvirus and periodontitis.

Herpesviruses cooperate with pathogenic bacteria in producing a variety of medical diseases, including hepatitis, encephalitis, otitis media with fever, infectious mononucleosis, Kaposi's sarcoma, oral hairy leukoplakia and dermal abscess, among many others [37-41] HCMV and EBV in particular, are two viruses which contribute to severe systemic diseases such as atherosclerosis, nasopharyngeal carcinoma, Burkitt's lymphoma, Non-Hodgkin's lymphoma, among others [37-41]. Since herpesvirus is associated with several systemic diseases, encouraging primary care practitioners to refer patients with herpesvirus for endodontic evaluation will lead to effective early diagnosis and possible treatment of periapical disease. Additionally, dentists working with physicians for the management of immunocompromised patients undergoing any type of organ transplant should monitor for any appearance of apical pathosis. 
The specific factors tied to the multifactorial etiological factors and pathogenesis of endodontic lesions will need broadening and/or review to consider the inclusion of herpesviruses among the other microorganisms and host immune responses.

Furthermore, herpesvirus-mediated immune suppression may be particularly destructive in granulomatous tissues because of an already compromised host immune response in periapical lesions.

\section{The Struggle between microbes and immunity}

Much about the etiopathogenesis of apical pathosis remains to be studied. The molecular events preceding disease onset and causative factors including the important roles of HCMV and EBV in the development of different periapical lesions need to be better understood. HCMV has been shown to increase A. actinomycetemcomitans adherence to epithelial cells providing evidence of the increased role of the virus with periodontitis [42]. There is a high probability that the combined presence of herpesviruses and endodontopathic bacteria increases the risk of a stable apical disease becoming unstable and developing an aggressive periapical and pulpal disease $[17,18]$. The intrinsic host impairment of endodontic lesions is likely to increase the risk of herpesvirus activation, establishing a vicious cycle. Furthermore, HCMV activation may trans-activate EBV. A high level of inflammatory cells co-infected with herpesvirus in apical lesions may generate favorable conditions for herpesvirus activation due to an immunocompromised host response $[17,18]$.

\section{The changing face of endodontics}

Among the many causative factors for apical pathosis, herpesvirus has emerged as a strong virulent link.

Severe periapical pathosis can be closely linked to herpesviral infection through the destructive immune responses that take place $[17,18]$ This may hold true in cases where absence of herpesvirus leads to an arrested state of apical breakdown. A strong causative link or correlation between herpesvirus and apical pathosis would set the stage for a new diagnosis method, better treatment modalities and efficacy. Such testing protocols would lead to more cost-effective treatment modalities by enabling earlier apical surgery and treatment, lowering costs in the future for low-income families.

A thorough criteria of endodontic lesions including progression of disease state, the role of herpesvirus, the onset of disease progression, and the delineation between acute and chronic apical pathosis is needed.

Through a classification like this, a diagnostic standard will be set using the latest research including viral vectors and disease progression. A better understanding of the complex interactions between herpesviruses, bacteria, other organisms, and host immune responses in periapical lesions might potentially lead to novel therapeutic approaches such as root canal irrigants with powerful antiviral action, antiviral medication, and vaccine development to prevent progression from unstable to stable apical pathosis. This would be an engaging and new first step in the link between herpesvirus and endodontic treatment. This could be further expanded by education for patients suffering from herpesvirus on their future need for endodontic treatment. By education, the overall systemic health of patients can be maintained and separate the line, not only between acute and chronic apical pathosis, but between medical and dental treatment needs, as well. Such criteria could be incorporated into medical and dental curricula, thereby increasing educational awareness between medical professionals. This would lead to better educational models for patients and address therapeutic needs.

\section{Conclusion}

Endodontic treatment needs for population-based decisions is unclear because of uncertainty over the prevalence of the herpesvirus and its impact on endodontic lesions, and the risk of disease progression. A one- time clinical assessment of pulp vitality and radiographic assessment is not sufficient for basic treatment needs. This is due to an unknown percentage of patients with herpesvirus infection who, in the absence of screening and subsequent treatment, would not experience uncomfortable tooth pain or tooth loss during their lifetime. The mechanism for a periapical lesion remaining unstable is yet not clear, though the latency of herpesviral vectors may play a part. This prompts a need for new treatment guidelines not just restricted to the pulp but also taking the inflammatory factors of herpesvirus into account. The criteria can be further applied to previous diagnosed cases of apical pathosis and set a new benchmark for diagnostic and treatment needs.

\section{Acknowledgement}

None.

\section{Conflict of Interest}

The authors do not disclose conflicts of interest.

\section{References}

1. Dahlen G (2002) Microbiology and treatment of dental abscesses and periodontal-endodontic lesions. Periodontal 2000 28(1): 206-239.

2. Gomes BP, Lilley JD, Drucker DB (1996) Associations of endodontic symptoms and signs with particular combinations of specific bacteria. Int Endod J 29(2): 69-75.

3. Sundqvist G (1992) Associations between microbial species in dental root canal infections. Oral Microbiol Immunol 7(5): 257-262.

4. Sabeti M, Slots J (2004) Herpesviral-bacterial coinfection in periapical pathosis. J Endod 30(2): 69-72.

5. Ferreira DC, Paiva SS, Carmo FL, Rocas IN, Rosado AS, et al. (2011) Identification of herpesviruses types 1 to 8 and human papillomavirus in acute apical abscesses. J Endod 37(1): 10-16.

6. Ferreira DC, Rocas IN, Paiva SS, Carmo FL, Cavalcante FS, et al. (2011) Viral-bacterial associations in acute apical disease. Oral Surg Oral Med Oral Pathol Oral Radiol Endod 112(2): 264-271.

7. Guilherme BP, Ferreira DC, Rocas IN, Provenzano JC, Santos KR, et al. (2011) Herpesvirus carriage in saliva and posttreatment apical periodontitis: searching for association. Oral Surg Oral Med Oral Pathol Oral Radiol Endod 112(5): 678-683.

8. Hernadi K, Szalmas A, Mogyorosi R, Czompa L, Veress G, et al. (2010) Prevalence and activity of Epstein-Barr virus and human cytomegalovirus in symptomatic and asymptomatic apical periodontitis lesions. J Endod 36(9): 1485-1489. 
9. Jakovljevic A, Andric M, Knezevic A, Soldatovic I, Nikolic N, et al. (2015) Human cytomegalovirus and Epstein-Barr virus genotypes in apical periodontitis lesions. J Endod 41(11): 1847-1851.

10. Slots J (2002) Chapter 16 Interactions between herpesviruses and bacteria in human periodontal disease. In Brogden KA, Guthmiller JM (Editors), Polymicrobial disesaes. ASM Press, Washington, DC, USA, pp. 317-331.

11. Hernadi K, Gyongyosi E, Meszaros B, Szakacs L, Szalmas A, et al. (2013) Elevated tumor necrosis factor-alpha expression in periapical lesions infected by Epstein-Barr virus. J Endod 39(4): 456-460.

12. Mogensen TH, Paludan SR (2001) Molecular pathways in virus-induced cytokine production. Microbiol Mol Biol Rev 65(1): 131-150.

13. Sabeti M, Simon JH, Slots J (2003) Cytomegalovirus and EpsteinBarr virus are associated with symptomatic periapical pathosis. Oral Microbiol Immunol 18(5): 327-328.

14. Rossini G, Cerboni C, Santoni A, Landini MP, Landolfo S, et al. (2012) Interplay between human cytomegalovirus and intrinsic/innate host responses: a complex bidirectional relationship. Mediators Inflamm 2012: 607276 .

15. Tortorella D, Gewurz BE, Furman MH, Schust DJ, Ploegh HL (2000) Viral subversion of the immune system. Annu Rev Immunol 18: 861-926.

16. Stern J, Shai E, Zaks B, Halabi A, Houri-Haddad Y, et al. (2004) Reduced expression of gamma interferon in serum and marked lymphoid depletion induced by Porphyromonas gingivalis increase murine morbidity and motality due to cytomegalovirus infection. Infect Immun 72(10): 5791-5798.

17. Sabeti M, Daneshmand A, Simon JH, Slots J (2009) Cytomegalovirusinfected inflammtory cells in dental periapical lesions. Oral Microbiol Immunol 24(5): 434-436.

18. Sabeti M, Kermani V, Sabeti S, Simon JH (2012) Significance of human cytomegalovirus and Epstein-Barr virus in inducing cytokine expression in periapical lesions. J Endod 38(1): 47-50.

19. American Association of Endodontists (2016) Glossary of endodontic terms. 8th edition. American Association of Endodontists, Chicago, IL, USA

20. Kirkevang LL, Vaeth M, Horsted-Bindslev P, Wenzel A (2006) Longitudinal study of periapical and endodontic status in a Danish population. Int Endod J 39(2): 100-107.

21. Jimenez-Pinzon A, Segura-Egea JJ, Poyato-Ferrera M, Velasco-Ortega E, Rios-Santos JV (2004) Prevalence of apical periodontitis and frequency of root-filled teeth in an adult Spanish population. Int Endod J 37(3): $167-173$

22. Dugas NN, Lawrence HP, Teplitsky PE, Pharoah MJ, Friedman S (2003) Periapical health and treatment quality assessment of root-filled teeth in two Canadian populations. Int Endod J 36(3): 181-192.

23. Siqueira JF Jr, Rocas IN (2005) Exploiting molecular methods to explore endodontic infections: Part 1-current molecular technologies for microbiological diagnosis. J Endod 31(6): 411-423.

24. Slots J (2005) Herpesviruses in periodontal diseases. Periodontol 2000 38: 33-62.

25. Gemmell E, Yamazaki K, Seymour GJ (2007) The role of T cells in periodontal disease: homeostasis and autoimmunity. Periodontol 2000 43: $14-40$.
26. Ting M, Contreras A, Slots J (2000) Herpesvirus in localized juvenile periodontitis. J Periodontal Res 35(1): 17-25.

27. Lofthag-Hansen S, Huumonen S, Grondahl K, Grondahl HG (2007) Limited cone-beam CT and intraoral radiography for the diagnosis of periapical pathology. Oral Surg Oral Med Oral Pathol Oral Radiol Endod 103(1): 114- 119.

28. Pigg M, List T, Petersson K, Lindh C, Petersson A (2011) Diagnostic yield of conventional radiographic and cone- beam computed tomographic images in patients with atypical odontalgia. Int Endod J 44(12): 10921101.

29. Verdugo F, Castillo A, Simonian K, Castillo F, Farez-Vidal E, et al. (2015) Periodontopathogen and Epstein-Barr virus-associated periapical periodontitis may be the source of retrograde infections peri-implantitis. Clin Implant Dent Relat Res 17(1): 199-207.

30. Chen CY, Hasselgren G, Serman N, Elkind MS, Desvarieux M, et al. (2007) Prevalence and quality of endodontic treatment in the Norther Manhattan elderly. J Endod 33(3): 230-234

31. Pinninti S, Hough-Telford C, Pati S, Boppana S (2016) Cytomegalovirus and Epstein-Barr virus infections. Pediatr Rev 37(6): 223-234.

32. Ozbas H, Asci S, Aydin Y (2011) Examination of the prevalence of periapical lesions and techincal quality of endodontic treatment in a Turkish subpopulation. Oral Surg Oral Med Oral Pathol Oral Radiol Endod 112(1):136-142.

33. Sissons JG, Carmichael AJ (2002) Clinical aspects and management of cytomegalovirus infection. J Infect 44(2): 78- 83.

34. Zaia JA, Forman SJ (1995) Cytomegalovirus infection in the bone marrow transplant recipient. Infect Dis Clin North Am 9(4): 879-900.

35. Aminoshariae A, Kulild JC, Mickel A, Fouad AF (2017) Association between systemic diseases and endodontic outcome: a systematic review. J Endod 43(4): 514-519.

36. Khalighinejad N, Aminoshariae MR, Aminoshariae A, Kulild JC, Mickel A, et al. (2016) Association between systemic diseases and apical periodontitis. J Endod 42(10): 1427-1434.

37. Dai L, DeFee MR, Cao Y, Wen J, Wen X, et al. (2014) Lipoteichoic acid (LTA) and lipopolysaccharides (LPS) from periodontal pathogenic bacteria facilitate oncogenic herpesvirus infection within primary oral cells. PLOS One 9(6): e101326.

38. Klein G (1989) Viral latency and transformation: the strategy of EpsteinBarr virus. Cell 58(1): 5-8.

39. Raab-Traub N, Rajadurai P, Flynn K, Lanier AP (1991) Epstein-Barr virus infection in carcinoma of the salivary gland. J Virol 65(12): 7032-7036.

40. Sixbey JW, Nedrud JG, Raab-Traub N, Hanes RA, Pagano JS (1984) Epstein-Barr virus replication in oropharyngeal epithelial cells. N Engl J Med 310(19): 1225-1230.

41. Tao Q, Ho FC, Loke SL, Srivastava G (1995) Epstein-Barr virus is localized in the tumour cells of nasal lymphomas of NK, T, or B cell type. Int J Cancer 60(3): 315-320.

42. Teughels W, Sliepen I, Quirynen M, Haake SK, Van Eldere J, et al. (2007) Human cytomegalovirus enhances A. actinomycetecomitans adherence to cells. J Dent Res 86(2): 175-180. 